\title{
Study on a Novel Pendulum Wave Energy Converter Combined of the Wind and Solar Energy
}

\author{
Qi Gao \\ North China Electric Power University, Beijing, 102206 \\ alanncepu@foxmail.com
}

Keywords: Clean energy; Pendulum wave energy converter; Intelligent monitoring

\begin{abstract}
There are much inexhaustible clean energy in the ocean. To generate electricity by using ocean energy can improve energy structure and be good for the environment. Existing wave energy converters have many disadvantages. So we designed a novel pendulum wave energy converter Combined of the Wind and Solar Energy, and pendulum wave energy converter is the core of it, which design conforms to the characteristics of the wave energy density in our country. It can collect wave energy through the floating plate and make full use of the coordination of the ratchet wheel and sprocket wheel to ensure one-way transmission of energy. Though more structural improvement, power generating efficiency of the device reach up to $48.96 \%$ and it has lower cost. We added solar power and wind power parts, which not only improve the overall output voltage, but also make fully use of clean energy of the sea. Furthermore, we designed the regulating circuit and control circuit to make data can be real-time display. The effect and feasibility of the device was verified through many experiments.
\end{abstract}

\section{The Background and Significance}

As a new energy, ocean energy has extensive sources and rare pollution. The potential nearshore ocean energy resources is about $15.8 \times 108 \mathrm{~kW}$ and the technical exploitation amount reaches $6.47 \times 108 \mathrm{~kW}$, showing that ocean energy has great prospect of development and utilization. The shortage of exploiting wave energy is that wave energy is affected by the monsoon which has short wind blowing time and small intensity. So the annual average power along the coast is only $3 \sim 7 \mathrm{~kW}$. Therefore we can conclude that large-scale grid-generation does not conform to the characteristics of wave energy power generation in China and independent power supply for ocean special site deserves us to study. Some existing device such as tail-first design, sea clams type and raft style which have complex construction, low power generating efficiency and high costing are can't resist extreme weather conditions. Above on, pendulum wave energy converter has good development prospects. The key of the converter is the design of its swing board and the way to achieve resonance. So we designed a novel pendulum wave energy converter. Considering that wave power is not stable and how to make full use of the renewable energy sources of the ocean, we designed our novel pendulum wave energy converter combined of the wind and solar energy.

\section{Designing Scheme of the Device}

General Design. Because of the low energy density of wave in our country and the shortage of existing device, we designed our novel pendulum wave energy converter. Although it has great generating efficiency and mechanical property, the voltage fluctuation is big for the wave on the sea is impressed by the weather and wave has height sinusoidal variation. Some energy with low voltage can't be used. To improve the capacity factor of pendulum wave energy converter and make full use of the luxuriant energy sources of the ocean, we designed our novel pendulum wave energy converter combined of the wind and solar energy.

Design of Control Circuit. Due to the power system's unstable output, power distribution reliability can't be satisfied and the storage battery will be damaged if we send the power directly to the load and storage battery. So we add the voltage regulator circuit and charging control circuit on our device. Using control module MCU, we can see the output voltage, the charging and 
discharging current of storage battery and its electric quantity on OLED in time.

Design of Mechanical Structure. The device is composed of energy harvesting, mechanical transmission and generating electricity. Firstly, waves can be converted into electrical energy and mechanical energy, and then the mechanical energy is changed into electricity. The overall structure design is shown as follows.

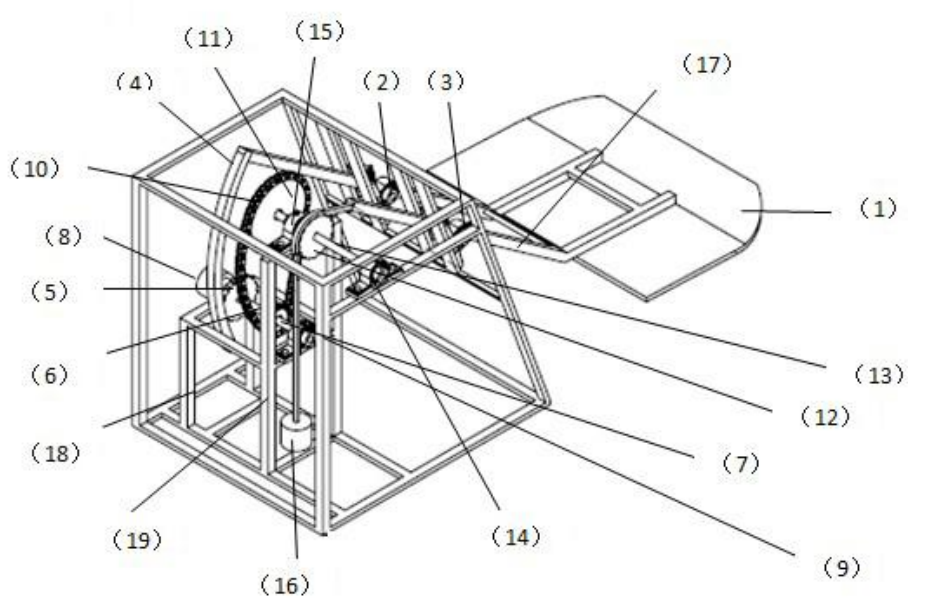

Figure 1. Design Diagram of the Converter

Notes:(1)floating plate (2)bearing A (3)bearing B (4)rack (5)ratchet wheel A (6)ratchet wheel B (7)fixed axis A (8)generator (9)bearing C (10)link chain (11)chain wheel (12)ratchet wheel (13)fixed axis B (14)bearing D (15)bearing E (16)mass (17) oscillating bar (18)device support A (19)device support B

From the picture above we can see that floating plate is connected to the oscillating bar which is fixed outside the walls of the device through bearing A and bearing B. The end of oscillating bar is connected to the rack. Rack and ratchet $\mathrm{A}$ bite. The ratchets wheel $\mathrm{A}$ and the ratchet wheel $\mathrm{B}$ coaxial fixed on the fixed axis $\mathrm{A}$. One end of fixed shaft $\mathrm{A}$ is connected to the generator and the other end fixed on the bearing $\mathrm{C}$. Bearing $\mathrm{C}$ is fixed on the square device support $\mathrm{A}$, ratchet $\mathrm{B}$ is connected to chain wheel through the chain $\mathrm{A}$. The chain wheel and the ratchet wheel $\mathrm{C}$ coaxial fixed on the fixed axis B. Both ends of rectangle fixed axis B is fixed on the device support B through the bearing D and the bearing $\mathrm{E}$. The mass is linked to oscillating bar through the chain A. The chain $\mathrm{B}$ and ratchet $\mathrm{C}$ bite. The device is fixed on the seabed. The outer wall of it is right-angle and send oscillating bar into the sea, making the floating plate and the sea surface fit closely together.

The design of size and power calculation of each part are as follows. Air resistance can be ignored in the calculation so we can think approximately that weight falling at a constant speed. The proportion of the lever is $K_{1}=R_{1}: R_{2}$.The proportion of the axle is $K_{2}=R_{3}: R_{4}$. The ratio of the side ratchet wheel and axle is $K_{31}=R_{5}: R_{6}$. The ratio of side ratchet wheel of gear rack and axle is $K_{32}=R_{5}: R_{7}$.( $R_{1}$ is radius of the front lever. $R_{2}$ is radius of the back-end lever. $R_{3}$ is radius of the ratchet hanging mass. $R_{4}$ is radius of the big chain wheel. ${ }^{R_{5}}$ is radius of the side ratchet wheel. ${ }^{6}$ is radius of the generator shaft. $R_{7}$ is radius of the side ratchet wheel of gear rack.)

To keep the balance of two lever and guarantee the security of the device, $K_{1} K_{2} K_{31}=K_{1} K_{32}$ must be satisfied. Buoyancy of Unit area of floating plate is $F$ and the area of floating plate is $S$,

so each lever is under the force $F^{\prime}=1 / 2 F S$. According to the wave cycle of different parts, we can calculate the angular velocity $\Omega$ of the lever. The power is:

$$
P=\frac{1}{2} \Omega F S K_{1} K_{2} K_{31} R_{1}+\frac{1}{2} \Omega F S K_{1} K_{32} R_{1}
$$


Taking the transfer efficiency $\eta$ into consideration, we know that the final power is $P^{\prime}=P \eta$.

\section{Theoretical Calculation}

According to the investigation and analysis of the wave and tide around the site location, we find that the annual average wave period is about $3.22 \mathrm{~s}$. However, it reaches about $4 \mathrm{~s}$ in summer. Therefore the average wave period of pendulum wave energy converter we designed should be between $1.9 \mathrm{~s}$ to $4.0 \mathrm{~s}$.

The Calculation of the Chain Length. Sprocket wheel and the ratchet wheel is linked through the chain, which size range is equal to the theory displacement range of floating plate. According to it, we can determine the length of the chain and calculate the rolling of float plate by the next formula:

$$
Z=\frac{\left(F_{0} / \rho g A_{w p}\right) \cos \left(\omega t+\gamma-\sigma_{z}\right)}{\sqrt{\left(1-\omega^{2} / \omega_{z}^{2}\right)^{2}+\left(2 \Delta_{z} \omega / \omega^{2}\right)^{2}}}=Z_{0} \cos \left(\omega t+\gamma-\sigma_{z}\right)
$$

Notes: ${ }^{F_{0}}$-the magnitude of the wave force; $Z_{0}$-motion amplitude; $t_{\text {-run duration }} \gamma_{\text {- phase }}$

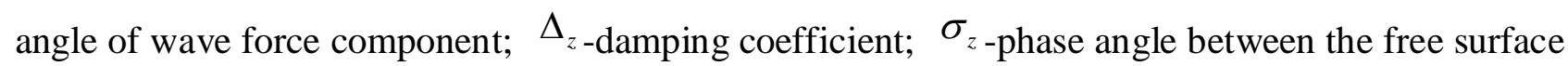
displacement and displacement of ups and downs $\omega_{\text {-wave circular frequency; } \rho \text {-density of sea }}$ water; $g$-acceleration of gravity $A_{w p}$-Floating board surface area; $\omega_{z-U p s}$ and downs natural angular frequency

After calculated, we find that floating plate amplitude $Z_{0}$ is $1.56 \mathrm{~m}$ and effective length of chain is to be at least $2 \mathrm{~m}$.

The Calculation of Conversion Efficiency. The schematic diagram of conversion efficiency is as follows.

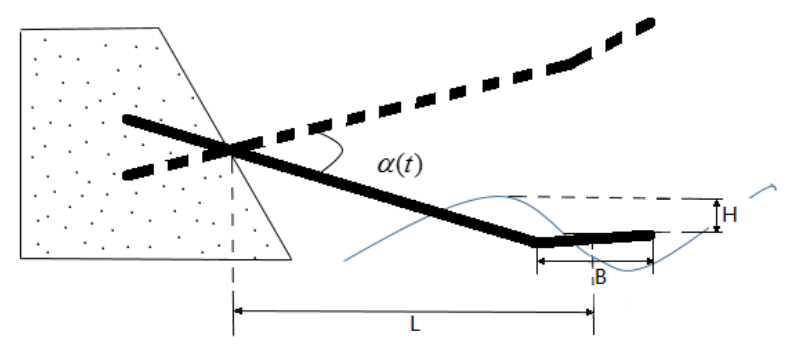

Figure 2. Diagram of the transfer efficiency

According to the diagram above, we can calculate the mechanical energy exporting by floating plate. Pendulum shaft torque is set to $M(t)$ and tilt angle of swing board is set to $\alpha(t)$.

Considering the overall structure and size of the device and the width of the floating plate, we can get the transfer efficiency of Oscillating floating plate is $\eta_{1}=0.593$.Furthermore, according to some relevant papers, we know that the transmission efficiency of gear is $96 \%$, the transmission efficiency of the chain is $97 \%$ and the transmission efficiency of bearing is $99 \%$. So if we make assumption that energy conversion efficiency of this part is $\eta_{2}=0.83$ and the efficiency of the generator is $\eta_{3}=0.96$, the final efficiency of the machine is $\eta=\eta_{1} \eta_{2} \eta_{3}=0.4896$.

\section{Analysis of Working Process and Performance}

We chose a sunny day with wind 3-4 to test. The device is put in the pool depth $40 \mathrm{~cm}$. When the waves be close to the device, the floating plate will have upward movement and drive lever to move with it. Rack, linked to the lever, could drive the ratchet wheel to move. And then, the generator will work. At the same time, the rising of floating plate will drive the chain of another ratchet wheel 
to lift the weight. In this way, the energy of waves can be converted into the gravitational potential energy of weight. When the waves go far away, the floating plate will fall and the device will work continuously in function of the weight. Analogously, the generator will generate electricity.

Meanwhile, solar panel and draught fan produce electric energy. All the electric energy output steadily as $12 \mathrm{~V}$ under the action of voltage regulator circuit. We measured the output voltage and charging voltage of storage battery. The test result proves the feasibility of device.

\section{The Innovation and Application Prospects}

Innovation. Though ingenious cooperation of ratchet wheel and sprocket and delicate design of floating plate, our novel pendulum wave energy converter has many advantages such as simple, efficient and having low cost, which is very suitable for the low wave energy density of our country.

Wave power generation is the main part of electric energy produced by the device and solar energy and wind power generation play a supporting role. In this way, renewable energy sources in the sea are fully used.

The design of CMOD of voltage stability and battery charging can provide steady voltage for battery. Information of battery charging and discharging can be real-time displayed and passed on PC for users to control.

All parts of the device use pollution free energy sources, so it set an example to exploitation of energy resources and possess good popularization and application.

Application Prospects. With so many advantages, the device conforms to the policy of developing green energy in our country. It could supply power for port, island and other near sea power equipment. As a replacement of small diesel engine, it could reduce the usage amount of diesel. Comforting to the trend of Internet+energy intelligence, the novel pendulum wave energy converter combined of the wind and solar energy has a broad market prospect.

\section{References}

[1] Wang Quanquan, Qin Chuan, Ju Ping, Wu Feng. 2014. A Coordinated Control Strategy for Hybrid Offshore Renewable Energy Power Generation Considering State of Charge of Battery Energy Storage System. Power System Technology: Vol. 38, No. 1, pp:80-86

[2] Chengzhi Yang, Siming Tan. 2014 The World Ocean Energy Patent Technology Analysis Report. Beijing: Ocean Press

[3] Qin Chuan, Wen Danyin, Ju Ping, Wu Feng. 2015. Comparison of models of hybrid offshore renewable energy generation system. Journal of Hohai University( Natural Sciences): Vol.3, No.6,pp:574-581

[4] Yongqiang Zhu. 2010 New Energy and Distributed Generation Technologies[M]. Beijing:Peking University Press

[5] QIN Chuan, JU Ping, WEN Danyin, WU Feng. 2012. Hybrid Offshore Wind, Wave and Tidal Turbine Energy Conversion System: Structure and Electrical Interface. Proceedings of the CSEE : Vol.34, No.5, pp.118-120

[6] Xiao Xi, Bai Nianzong, Kang Qing, Nie Zangxiang, Huang Xuanrui. 2014.A Review of the Development of Wave Power System and the Research on Direct-Drive Wave Power System. Institution of Telecommunication Satellite China Academy of Space Technology, Vol.29

[7] Chunyan Zhang. 2011. Research of Wave and Solar Energy Complementary Standalone Power Generation System[D]. Tianjin University

[8] Yang Zhao. 2015. Research on the Operation and Control of Energy Storage Device in Marine Hybrid Multiple Renewable Energy Power System on Island[D]. Northeast Normal University 
[9] Caixia Xue. Research on Control Technology of Multi-energy Hybrid Isolated Power System Based on Ocean Energy[D].National Ocean Technology Center

[10]Zhaocai Wang. 2012. Control Strategy of Intermittent Renewable Generation System and Application Research. Zhengzhou University 